Pacific Journal of Mathematics

SECOND ORDER DIFFERENTIAL OPERATORS WITH 


\title{
SECOND ORDER DIFFERENTIAL OPERATORS WITH SELF-ADJOINT EXTENSIONS
}

\author{
Arnold L. Villone
}

Let $\mathscr{H}$ denote the Hilbert space of square summable analytic functions on the unit disk, and consider those formal differential operators

$$
L=p_{2} \frac{d^{2}}{d z^{2}}+p_{1} \frac{d}{d z}+p_{0}
$$

which give rise to symmetric operators in $\mathscr{H}$. Examples have been given where the symmetric operators associated with these formal operators have defect indices $(0,0)$ and $(2,2)$ and hence are either self-adjoint or have self-adjoint extensions in $\mathscr{H}$. In this note a class of symmetric operators with defect indices $(1,1)$ is given.

Let $\mathscr{A}$ denote the space of functions analytic on the unit disk and $\mathscr{H}$ the subspace of square summable functions in $\mathscr{A}$ with inner product

$$
(f, g)=\iint_{|z|<1} f(z) \overline{g(z)} d x d y
$$

A complete orthonormal set for $\mathscr{H}$ is obtained by normalizing the powers of $z$. From this it follows that $\mathscr{H}$ is identical with the space of power series $\sum_{n=0}^{\infty} a_{n} z^{n}$ which satisfy

$$
\sum_{n=0}^{\infty}\left|a_{n}\right|^{2} /(n+1)<\infty
$$

Let $L$ be such that it maps polynomials into $\mathscr{H}$ and has the property $\left(L z^{n}, z^{m}\right)=\left(z^{n}, L z^{m}\right), n, m=\theta, 1,2, \cdots$. Let $\mathscr{D}_{0}$ be the subspace of polynomials and set $T_{0} f=L f$ for $f$ in $\mathscr{D}_{0}$. Then $T_{0}$ is symmetric and the defect indices $\mathrm{m}^{+}$and $\mathrm{m}^{-}$of its closure, $S$, are just the number of linearly independent solutions of $L u=i u$ and $L u=-i u$ respectively which are in $\mathscr{H}$. See [2]. In [2] and [3] examples of such symmetric operators $S$ with defect indices $(0,0)$ and $(2,2)$ are provided. We now give a class of operators with defect indices $(1,1)$. 


\section{Consider the operator $L$,}

$$
L=\left(c_{1} z^{3}+\bar{c}_{1} z\right) \frac{d^{2}}{d z^{2}}+\left(\left(c_{2}+3 c_{1}\right) z^{2}+\bar{c}_{2}\right) \frac{d}{d z}+2 c_{2} z .
$$

In [3] it is shown that $L$ gives rise to symmetric $T_{0}$. Concerning the defect indices of its closure $S$, we have the following.

THEOREM 2.1. Let $L$ be the operator of (2.1) then $S$ has defect indices $m^{+}=m^{-}=1$.

Proof. The idea of the proof is to show that the equation $L \phi=$ $\pm i \phi$ has precisely one power series solution $\phi(z)=\sum_{j=0}^{\infty} a_{j} z^{j}$ and that there exists a $K>0$ and a positive integer $p$ such that $\left|a_{j}\right| \leqq K j^{-1 / p}$ for $j$ sufficiently large. Consequently the series $\sum_{j=0}^{\infty}\left|a_{j}\right|^{2} /(j+1)$ converges and $\phi$ belongs to $\mathscr{H}$, and $m^{+}=m^{-}=1$.

Dividing $L \phi= \pm i \phi$ by $c_{1}$ we have the differential equation

$$
\left(z^{3}+\omega z\right) \phi^{\prime \prime}+\left[(3+\alpha) z^{2}+\beta\right] \phi^{\prime}+2 \alpha z \phi=\lambda \phi,
$$

where $\omega=\bar{c}_{1} / c_{1}, \alpha=c_{2} / c_{1}, \beta=\bar{c}_{2} / c_{1}$, and $\lambda= \pm i / c_{1}$.

Substituting $\sum_{j=0}^{\infty} a_{j} z^{j}$ into (2.2) we obtain

$$
\begin{gathered}
\beta a_{1}+\sum_{j=1}^{\infty}\left[(j+1)(\omega j+\beta) a_{j+1}+\left(j^{2}+j \alpha+\alpha-1\right) a_{j-1}\right] z^{j} \\
=\lambda a_{0}+\sum_{j=1}^{\infty} \lambda a_{j} z^{j} \quad \lambda \neq 0 .
\end{gathered}
$$

If $\beta=0$ we have $a_{0}=0$ and (2.3) can be solved recursively for $a_{2}, a_{3}, \cdots$, in terms of $a_{1}$ since $\omega j+\beta$ never vanishes. Thus we have but one analytic solution

$$
\phi(z)=z\left(1+a_{2} z^{2}+\cdots\right) .
$$

If $\beta \neq 0$, we have $a_{1}=\lambda a_{0} / \beta$ and (2.3) can be solved recursively for $a_{2}, a_{3}$, etc., provided that $(\omega j+\beta)$ never vanishes for $j=$ $1,2, \cdots$. Thus we are able to obtain the single formal power series solution $\phi(z)=1+a_{1} z+a_{2} z^{2}+\cdots$. The case when $(\omega j+\beta)$ vanishes for some positive integer $j$ presents some complications and will be considered later in the proof. Solving (2.3) for $a_{j+1}$ we have

$$
a_{j+1}=\frac{1}{\omega}\left\{\frac{-\left[j^{2}+j \alpha+(\alpha-1)\right] a_{j-1}+\lambda a_{j}}{j^{2}+\left(1+\frac{\beta}{\omega}\right) j+\frac{\beta}{\omega}}\right\} .
$$


But $\beta / \omega=\bar{c}_{2} / \bar{c}_{1}=\bar{\alpha}$, hence (2.4) becomes

$$
a_{j+1}=\frac{1}{\omega}\left\{\frac{-\left[j^{2}+j \alpha+(\alpha-1)\right] a_{j-1}+\lambda a_{j}}{j^{2}+(1+\bar{\alpha}) j+\bar{\alpha}}\right\} .
$$

Thus we obtain the estimate

$$
\begin{aligned}
\left|a_{j+1}\right| & \leqq \frac{1}{|\omega|}\left|\frac{j^{2}+j \alpha+(\alpha-1)}{j^{2}+(1+\bar{\alpha}) j+\bar{\alpha}}\right|\left|a_{j-1}\right| \\
& +\frac{|\lambda|}{|\omega|} \frac{1}{\left|j^{2}+(1+\bar{\alpha}) j+\bar{\alpha}\right|}\left|a_{j}\right| .
\end{aligned}
$$

Since $|\omega|=1$ we have

$$
\left|a_{j+1}\right| \leqq\left|u_{1}(j)\right|\left|a_{j-1}\right|+\left|u_{2}(j)\right|\left|a_{j}\right|
$$

where

$$
u_{1}(j)=\frac{j^{2}+j \alpha+(\alpha-1)}{j^{2}+(1+\bar{\alpha}) j+\bar{\alpha}}
$$

and

$$
u_{2}(j)=\frac{\lambda}{j^{2}+(1+\bar{\alpha}) j+\bar{\alpha}}
$$

We now estimate $\left|u_{1}(j)\right|$ and $\left|u_{2}(j)\right|$ for large $j$. Since $\left|u_{2}(j)\right|$ tends to zero as $j^{-2}$ it follows that there exists an $M>0$ such that

$$
\left|u_{2}(j)\right| \leqq \frac{M}{j^{2}}, \quad \text { for } j \text { sufficiently large. }
$$

Concerning $\left|u_{1}(j)\right|$ we obtain, upon dividing,

$$
u_{1}(j)=\left(1-\frac{1}{j}\right)+\frac{2}{j} \operatorname{Im}(\alpha) i+O\left(j^{-2}\right) .
$$

Thus $\left|u_{1}(j)\right|^{2}=1-2 / j+O\left(j^{-2}\right)$, and hence by a direct calculation,

$$
\left|u_{1}(j)\right|=1-\frac{1}{j}+O\left(j^{-2}\right)
$$

For $\xi>0$, we note that $\left|u_{1}(j)\right| \leqq 1-\xi j^{-1}$ for $j$ sufficiently large if and only if $-1<-\xi$, or $\xi<1$. Hence we have 


$$
\begin{aligned}
& \left|u_{1}(j)\right| \leqq 1-\frac{\xi}{j}, \text { for } j \text { sufficiently large } \\
& \text { and } 0<\xi<1 \text {. }
\end{aligned}
$$

Using (2.6), (2.7), and (2.8) we obtain, for $j$ sufficiently large,

$$
\begin{aligned}
\left|a_{j+1}\right| & \leqq\left(1-\xi j^{-1}\right)\left|a_{j-1}\right|+M j^{-2}|| a_{j} \mid \\
& \leqq\left(1-\xi j^{-1}+M j^{-2}\right) M(j), \quad 0<\xi<1,
\end{aligned}
$$

where $M(j)=\max \left\{\left|a_{\jmath-1}\right|,\left|a_{j}\right|\right\}$.

Thus, for sufficiently large $j$, we have

$$
\left|a_{j+1}\right| \leqq\left(1-\gamma j^{-1}\right) M(j),
$$

where $0<\gamma=\xi / 2<\frac{1}{2}$.

Now consider the expression $\left(1-\gamma j^{-1}\right)(j-1)^{-1 / p}$, where $p$ is a positive integer. This is dominated by $(j+1)^{-1 / p}$ for $j$ sufficiently large if and only if

$$
j^{p+1}+(-p \gamma+1) j^{p}+\cdots \leqq j^{p+1}-j^{p}
$$

Hence, if and only if $-p \gamma+1<-1$ or $-p \gamma<-2$. Since $\gamma>0$, $p>2 / \gamma$. Thus we have

$$
\left(1-\gamma j^{-1}\right)(j-1)^{-1 / p} \leqq(j+1)^{-1 / p}, \quad p>\frac{2}{\gamma}
$$

We now show that there exists a positive constant $K$ for which $\left|a_{j}\right| \leqq K j^{-1 / p}$ for $j \geqq 1$. Let $j_{1}$ be such that (2.9) and (2.10) hold for $j>j_{1}$. Let $K=\max _{j \leqq j_{1}}\left|a_{j}\right| j^{1 / p}$ so that $\left|a_{j}\right| \leqq K j^{-1 / p}$ for $j \leqq j_{1}$. Using (2.9) it follows that

$$
\left|a_{j_{1}+1}\right| \leqq\left(1-\gamma j_{1}^{-1}\right) M\left(j_{1}\right)
$$

where

$$
\begin{aligned}
M\left(j_{1}\right) & =\operatorname{Max}\left(K j_{1}^{-1 / p}, K\left(j_{1}-1\right)^{-1 / p}\right) \\
& =K\left(j_{1}-1\right)^{-1 / p} .
\end{aligned}
$$

Hence,

$$
\left|a_{j_{1}+1}\right| \leqq\left(1-\gamma j_{1}^{-1}\right) K\left(j_{1}-1\right)^{-1 / p}
$$


and using (2.10) we have

$$
\left|a_{j+1}\right| \leqq K\left(j_{1}+1\right)^{-1 / p}
$$

We now proceed inductively to establish

$$
\left|a_{j_{1}+k}\right| \leqq K\left(j_{1}+k\right)^{-1 / p}, \quad k=2,3, \cdots
$$

Let

$$
\begin{aligned}
K_{1} & =\max _{j \leqq j_{1}+1}\left|a_{j}\right| j^{1 / p} \\
& =\max \left\{K, K\left(j_{1}+1\right)^{-1 / p}\right\} \leqq K,
\end{aligned}
$$

making use of (2.11). Using (2.9) we have

$$
\left|a_{j_{1}+2}\right| \leqq\left(1-\gamma\left(j_{1}+1\right)^{-1} M\left(j_{1}+1\right),\right.
$$

where,

$$
\begin{aligned}
M\left(j_{1}+1\right) & =\operatorname{Max}\left(\left|a_{j_{1}+1}\right|,\left|a_{j_{1}}\right|\right) \\
& =\operatorname{Max}\left(K\left(j_{1}+1\right)^{-1 / p}, K\left(j_{1}\right)^{-1 / p}\right) \\
& =K\left(j_{1}\right)^{-1 / p} .
\end{aligned}
$$

It follows from (2.10) that

$$
\begin{aligned}
\left|a_{j_{1}+2}\right| & \leqq\left(1-\gamma\left(j_{1}+1\right)^{-1}\right) K\left(j_{1}\right)^{-1 / p} \\
& \leqq K\left(j_{1}+2\right)^{-1 / p} .
\end{aligned}
$$

Continuing on in this manner we establish (2.12). Hence any solution $\sum_{j=0}^{\infty} a_{j} z^{j}$ whose coefficients satisfy (2.4) is in $\mathscr{H}$. To complete the proof we have only to deal with the case where $j \omega+\beta$ vanishes for some positive integer $j$.

We now consider the case when $j \omega+\beta$ vanishes for some positive integer $n$. The analytic solution obtained from (2.3) by taking $a_{0}=a_{1}=$ $\cdots=a_{n}=0$, and solving recursively for $a_{n+2}, a_{n+3}, \cdots$, in terms of $a_{n+1}$ is, as we have seen, in $\mathscr{H}$. If there were a second analytic solution corresponding to $a_{0} \neq 0$ it would be in $\mathscr{H}$ as well, and $m^{+}\left(m^{-}\right)$would be 2. We now show that this is not the case, i.e., $m^{+}=m^{-}=1$. To do this we make use of the following result.

Let $\mu$ be such that $\operatorname{Im}(\mu)>0$ and let $\mathscr{D}_{\mu}^{+}$be the nullspace of the operator $S^{*}-\mu$. Then the dimension of $\mathscr{D}_{\mu}^{+}$is equal to $m^{+}$. Similarly, 
let $\operatorname{Im}(\mu)<0$ and let $\mathscr{D}_{\mu}^{-}$be the nullspace of the operator $S^{*}-\mu$, then the dimension of $\mathscr{D}_{\mu}^{-}$is equal to $m^{-},[1$, p. 1232].

Using this we see that $m^{+}$is just the number of linearly independent solutions of $L \phi=\mu \phi$ in $\mathscr{H}$ for any $\mu$ such that $\operatorname{Im}(\mu)>0$. Similarly, $m^{-}$is the number of linearly independent solutions of $L \phi=\mu \phi$ in $\mathscr{H}$ for any $\mu$ such that $\operatorname{Im}(\mu)<0$. Hence, if we can show that there exist $\mu$ such that $\operatorname{Im} \mu>0(\operatorname{Im} \mu<0)$ for which there is no analytic solution corresponding to $a_{0} \neq 0$ we will have shown that $m^{+}=m^{-}=1$.

Consider (2.3), where $\lambda$ is now $\mu / c_{2}$, and suppose that $\beta=$ $-n \omega$. Taking $j=1,2, \cdots, n$ we obtain the following set of $n+1$ linear equations in $a_{0}$ thru $a_{n}$ :

$$
\begin{aligned}
& -n \omega a_{1}=\lambda a_{0} \\
& \begin{aligned}
(j+1)(j-n) \omega a_{j+1}+\left(j^{2}+j \alpha+\alpha-1\right) a_{j-1} & =\lambda a_{j}, \\
j=1,2, \cdots, n-1 & \\
\left(n^{2}+n \alpha+\alpha-1\right) a_{n-1} & =\lambda a_{n} .
\end{aligned}
\end{aligned}
$$

Thus we are led to consider the homogeneous system

$$
\begin{aligned}
-\lambda a_{0}-n \omega a_{1} & =0 \\
2 \alpha a_{0}-\lambda a_{1}+2(2-n) \omega a_{2} & =0 \\
\left(n^{2}+n \alpha-2 n\right) a_{n-2}-\lambda a_{n-1}-n \omega a_{n} & =0 \\
\left(n^{2}+n \alpha+\alpha-1\right) a_{n-1}-\lambda a_{n} & =0
\end{aligned}
$$

Since the parameter $\lambda=\mu / c_{2}$ appears only on the diagonal the system determinant $D_{n}(\lambda)$ is a polynomial in $\lambda$ of degree $n+1$,

$$
D_{n}(\lambda)=(-1)^{n+1} \lambda^{n+1}+\cdots .
$$

Thus $D_{n}(\lambda)$ vanishes at most $n+1$ points in the complex plane, and we can find $\mu$ in the upper half-plane and lower half-plane for which $D_{n}\left(\mu / c_{2}\right) \neq 0$. Thus $a_{0}=a_{1}=\cdots=a_{n}=0$ and there is only one analytic solution of $L \phi=\mu \phi$.

\section{REFERENCES}

1. N. Dunford and J. Schwartz, Linear Operators, Part II, Interscience Publishers, 1963.

2. A. L. Villone, Self-adjoint differential operators, Pacific J. Math., 35 (1970), 517-531.

3. Self-Adjoint Extensions of Symmetric Differential Operators, Pacific J. Math., 49 (1974), 569-577. 


\section{CONTENTS}

Zvi Artstein and John A. Burns, Integration of compact set-valued functions

J. A. Beachy and W. D. Blair, Rings whose faithful left ideals are cofaithful

Mark Benard, Characters and Schur indices of the unitary reflection group $[321]^{3}$

H. L. Bentley and B. J. Taylor, Wallman rings ............................. 15

E. Berman, Matrix rings over polynomial identity rings II ............... 37

Simeon M. Berman, A new characterization of characteristic functions of absolutely continuous distributions ........................ 323

Monte B. Boisen, Jr. and Philip B. Sheldon, Pre-Prüfer rings ............ 331

A. K. Boyle and K. R. Goodearl, Rings over which certain modules are injective

J. L. Brenner, R. M. Crabwell and J. Riddell, Covering theorems for finite nonabelian simple groups. $V$

H. H. Brungs, Three questions on duo rings .................................... 345

Iracema M. Bund, Birnbaum-Orlicz spaces of functions on groups ....351

John D. Elwin and Donald R. Short, Branched immersions between 2-manifolds of higher topological type

J. K. Finch, The single valued extension property on a Banach space ................................................................................. 61

J. R. Fisher, A Goldie theorem for differentiably prime rings ............ 71

Eric M. Friedlander, Extension functions for rank 2, torsion free abelian groups ...................................................................... 371

J. Froemke and R. Quackenbusch, The spectrum of an equational class of groupoids

B. J. Gardner, Radicals of supplementary semilattice sums of

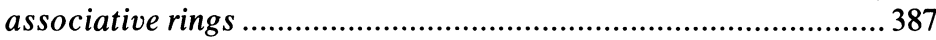

Shmuel Glasner, Relatively invariant measures ...............................393

G. R. Gordh, Jr. and Sibe Mardešić, Characterizing local connectedness in inverse limits...

S. Graf, On the existence of strong liftings in second countable topological spaces

S. Gudder and D. Strawther, Orthogonally additive and orthogonally increasing functions on vector spaces ........................................427

F. Hansen, On one-sided prime ideals .......................................... 79

D. J. Hartfiel and C. J. Maxson, A characterization of the maximal monoids and maximal groups in $\beta x$.

Robert E. Hartwig and S. Brent Morris, The universal flip matrix and the generalized faro-shuffle 


\section{Pacific Journal of Mathematics}

Vol. 58, No. 1

March, 1975

John Allen Beachy and William David Blair, Rings whose faithful left ideals are cofaithful .................................... 1

Herschel Lamar Bentley and Barbara June Taylor, Wallman rings ........ 15

Elizabeth Berman, Matrix rings over polynomial identity rings. II ...... 37

Ann K. Boyle and Kenneth R. Goodearl, Rings over which certain modules are injective ................................. 43

J. L. Brenner, Robert Myrl Cranwell and James Riddell, Covering theorems for finite nonabelian simple groups. $V \ldots \ldots \ldots \ldots \ldots \ldots \ldots \ldots \ldots$

James Kenneth Finch, The single valued extension property on a Banach

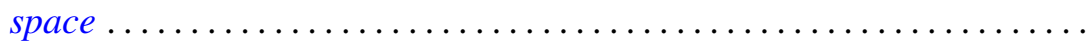

John Robert Fisher, A Goldie theorem for differentiably prime rings........ Friedhelm Hansen, On one-sided prime ideals .................... Jon Craig Helton, Product integrals and the solution of integral equations..........................................

Barry E. Johnson and James Patrick Williams, The range of a normal

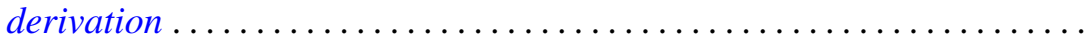

Kurt Kreith, A dynamical criterion for conjugate points ............ 123

Robert Allen McCoy, Baire spaces and hyperspaces .............. 133

John McDonald, Isometries of the disk algebra ................ 143

H. Minc, Doubly stochastic matrices with minimal permanents ......... 155

Shahbaz Noorvash, Covering the vertices of a graph by vertex-disjoint paths. ...

Theodore Windle Palmer, Jordan *-homomorphisms between reduced Banach*-algebras

Donald Steven Passman, On the semisimplicity of group rings of some

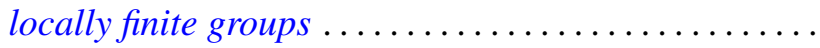

Mario Petrich, Varieties of orthodox bands of groups .

Robert Horace Redfield, The generalized interval topology on distributive

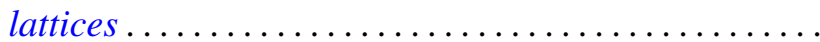

James Wilson Stepp, Algebraic maximal semilattices .... . .

Patrick Noble Stewart, A sheaf theoretic representation of rings with Boolean orthogonalities ........................

Ting-On To and Kai Wing Yip, A generalized Jensen's inequality......... 255

Arnold Lewis Villone, Second order differential operators with self-adjoint

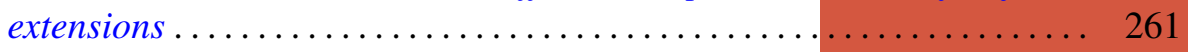

Martin E. Walter, On the structure of the Fourier-Stieltjes algebra ....... 267

John Wermer, Subharmonicity and hulls .................... 283

Edythe Parker Woodruff, A map of $E^{3}$ onto $E^{3}$ taking no disk onto a

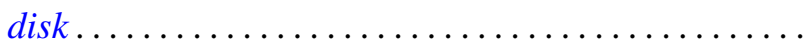

